\title{
Society Bulletins
}

\section{Arzneimittelengpässe in der Onkologie. Management verbessert - Bedrohung bleibt}

Auch in diesem Jahr sind Lieferengpässe bei Arzneimitteln in Deutschland ein großes Thema. Zwar sind wirkliche Versorgungsengpässe weiterhin selten, die Verunsicherung bei PatientInnen, ÄrztInnen und ApothekerInnen ist aber hoch. Die Engpässe dieses Sommers haben auch die Onkologie betroffen. Bei vielen KrebspatientInnen ist die medikamentöse Tumortherapie ein zentrales Element der Behandlung. Von der Verfügbarkeit eines Arzneimittels kann die Überlebenschance abhängen. Die DGHO Deutsche Gesellschaft für Hämatologie und Medizinische Onkologie e. V. hatte bereits im März 2017 einen Band ihrer Gesundheitspolitischen Schriftenreihe zu der Thematik herausgegeben. Nun diskutiert sie aufgrund der weiter bestehenden Probleme, welche weiteren Schritte zur Sicherung erforderlich und sinnvoll sind.

Arzneimittelengpässe sind ein weltweites Problem. Die Ursachen sind vielfältig, die Auswirkungen sind in jedem Land unterschiedlich. Zwar muss sich nicht jeder Lieferengpass zwingend zu einem Versorgungsengpass entwickeln, wenn es aber zu Schwierigkeiten bei der Versorgung kommt, kann es sowohl bei Erwachsenen als auch bei Kindern zur Verschlechterung der Prognose führen. Dass Arzneimittelengpässe seit Jahren ein Problem sind, betonte Prof. Dr. med. Michael Hallek Geschäftsführender Vorsitzender der DGHO und Direktor der Inneren Medizin I - Onkologie, Hämatologie, Klinische Infektiologie, Klinische Immunologie, Hämostaseologie, Internistische Intensivmedizin an der Universitätsklinik Köln und des Centrums für Integrierte Onkologie (CIO) Köln Bonn: „Wir stellen fest, dass die Anzahl der Lieferengpässe von Arzneimitteln in den vergangenen Jahren leider zugenommen hat. Das ist völlig inakzeptabel. Dass sich Lieferengpässe nicht automatisch zu Versorgungsengpässen entwickeln, haben wir nur einer gemeinsamen Kraftanstrengung verschiedener Akteure zu verdanken."

\section{Erste wichtige Schritte sind gemacht}

Ein erster wichtiger Schritt hin zu mehr Transparenz und verbessertem Informationsfluss, so Prof. Dr. med. Karl Broich, Präsident des Bundesinstituts für Arzneimittel und Medizinprodukte (BfArM), war die Implementierung eines Jour Fixe im Jahr 2016. „Durch die gute Zusammenarbeit im Jour Fixe können wir im engen Austausch mit den medizinischen Fachgesellschaften und der pharmazeutischen Industrie besondere Problemlagen rasch identifizieren und mögliche Lösungswege für die Versorgungssicherheit der Patientinnen und Patienten anstoßen. Deshalb begrüßen wir ausdrücklich, dass unsere Handlungsmöglichkeiten im Sinne des Patientenschutzes künftig weiter ausgebaut werden."

\section{Frühzeitige Einbindung von Fachgesellschaften}

Den Aspekt des Versorgungsmanagements verdeutlichte Hallek: „Am Beispiel des Lieferengpasses von Etopophos im Jahr 2016 haben wir gelernt, wie wichtig die frühzeitige Einbindung der wissenschaftlichen medizinischen Fachgesellschaften und anderer betroffener Akteure ist. Unmittelbar nach Bekanntwerden der geplanten Kontingentierung von Etopophos haben wir gemeinsam 
mit der Gesellschaft für Pädiatrische Onkologie und Hämatologie $(\mathrm{GPOH})$ und dem Bundesverband Deutscher Krankenhausapotheker (ADKA) Empfehlungen erarbeitet, die die Versorgung von Patientinnen und Patienten mit Etopophos, bei denen aus medizinischen Gründen nicht auf das alkohollösliche Etoposid zurückgegriffen werden kann, sicherstellen konnten."

\section{Apotheken müssen entlastet werden}

Die Sicht der ApothekerInnen verdeutlichte Prof. Dr. rer. nat. Martin Schulz, Vorsitzender der Arzneimittelkommission der Deutschen Apotheker (AMK), Berlin: „Die Problematik der Lieferengpässe hat sich in den letzten Jahren deutlich verschärft. Von den etwa 450 Millionen Rabattarzneimitteln in der GKV waren 2017 ca. 4,7 Millionen nicht lieferfähig. 2018 waren es 9,3 Millionen. Das ist eine Verdoppelung! Jedes 50. rabattfähige Arzneimittel war betroffen. Dieser Trend hält mit 7,2 Millionen Packungen allein im ersten Halbjahr 2019 an. "Untersuchungen des Deutschen Arzneiprüfungsinstituts e.V. (DAPI) zeigten, dass mengenmäßig vor allem Schmerzmittel, Blutdrucksenker und Antidepressiva betroffen waren. Für die Apotheken bedeutet dies nach Ansicht von Schulz vor allem viel Arbeit: „60\% wenden mindestens 10\% ihrer Arbeitszeit dafür auf, Lieferengpässe zu managen. Neun von zehn ApothekenleiterInnen sagen, dass Lieferengpässe das größte Ärgernis in ihrem Arbeitsalltag sind. Wir wissen aus einer Umfrage bei den AMK-Referenzapotheken, dass Apotheken trotz dieser Hindernisse stets versuchen, die adäquate Arzneimittelversorgung in Deutschland sicherzustellen und damit die Patientensicherheit zu wahren. Dies bedarf hoher fachlicher Expertise, interprofessioneller Zusammenarbeit und einer intensiven Beratung der PatientInnen, denn nicht immer steht ein Arzneimittel eines anderen Herstellers mit dem identischen Wirkstoff als vollwertige Alternative zur Verfügung. Dann muss beispielsweise Rücksprache mit dem verschreibenden Arzt zwecks einer Alternativverordnung gehalten werden, oder die Versorgung des Patienten wird durch Anfertigung eines Rezepturarzneimittels sichergestellt." Letzteres bestätigten laut Schulz über 55\% der befragten Krankenhausapotheken und knapp 20\% der öffentlichen Apotheken. Schulz ergänzte: „Wir fordern seit langem, dass Probleme in der Lieferkette vor allem frühzeitig erkannt und kommuniziert werden müssen. Es bedarf jetzt einer gesetzlichen Meldepflicht für pharmazeutische Unternehmen, umfassende Informationen allen Beteiligten schnellstmöglich zur Verfügung zu stellen, damit Apotheken rechtzeitig Gegenmaßnahmen ergreifen können."

\section{DGHO fordert konkrete Maßnahmen}

Mit Blick auf die von Schulz angesprochenen weiterhin bestehenden Defizite erläuterte Prof. Dr. med. Bernhard Wörmann, Medizinischer Leiter der DGHO, die in der von der DGHO vorgelegten Aktualisierung enthaltenden Forderungen zur Vermeidung von Liefer- und Versorgungsengpässen. Diese sind u.a.:

- Register mit Meldepflicht: Ein Register ist (in der Onkologie) nur brauchbar, wenn es zuverlässig über alle Arzneimittel einschließlich der supportiven Therapie Auskunft gibt. Die DGHO fordert ein verpflichtendes Register für alle Lieferengpässe.

- Schutz unverzichtbarer Arzneimittel: Im März 2019 hat sich der Jour Fixe im Positionspapier zu „Lieferund Versorgungsengpässen beim BfArM zur Vertragsgestaltung zwischen Kliniken und pharmazeutischen Unternehmen mit dem Ziel einer Verbesserung der Lieferfähigkeit versorgungsrelevanter Basistherapeutika in Kliniken" auf Kriterien versorgungskritischer Lieferengpässe verständigt. Wirksame Maßnahmen zur Umsetzung der Forderungen für die Onkologie sind:

- Vorratshaltung

- Verträge mit langfristiger Liefersicherheit

- Förderung qualitativ hochwertiger Herstellung von Arzneimitteln: Das BfArM ging 2016 davon aus, dass 90\% der Lieferengpässe bei Arzneimitteln durch Qualitätsmängel in der Produktion bedingt sind. Qualitätsmängel können in allen Produktionsstätten auftreten. Die DGHO fordert die Förderung und die zuverlässige Überwachung von Produktionsstätten nach EU-Standard.

\section{Politik ist gefordert}

Dass das Thema von Liefer- und Versorgungsengpässen auch in der politischen Debatte angekommen ist, machte Michael Hennrich (CDU), Mitglied im Bundestag und Obmann im Ausschuss für Gesundheit, deutlich: „Patientinnen und Patienten sind zurecht beunruhigt, wenn ihr gewohntes Arzneimittel in der Apotheke nicht erhältlich ist. Daher müssen wir Lieferengpässen von Medikamenten nachhaltiger vorbeugen und eine dauerhaft zuverlässige Versorgung mit sicheren Arzneimitteln gewährleisten." Mit dem jüngst verabschiedeten Gesetz für mehr Sicherheit in der Arzneimittelversorgung (GSAV) hat der Gesetzgeber zwar verschiedene Maßnahmen verankert. Darüber hinaus sind aber eine ganze Reihe von weitergehenden Schritten notwendig. Dazu gehörten, so Hennrich, Transparenz über das Liefer- und Marktgeschehen, verbindliche Meldepflichten, längere Vorratshaltung, Exportbeschränkung im Falle bestehender Lie- 
ferengpässe, Anpassung der Ausschreibungs- und Vergabemodalitäten und Erhöhung der Marktchancen und damit den Absatz für Arzneimittel „Made in Europe“.

\section{Trotz aller positiven Entwicklungen: Die Bedrohung bleibt}

Zum Abschluss der Pressekonferenz betonte Hallek, dass das Thema von Liefer- und Versorgungsengpässen trotz aller positiven Entwicklungen nichts an Brisanz und Dramatik eingebüßt hat. „Wir haben es in den vergangenen Jahren leider viel zu oft erlebt, dass wir als Ärztinnen und Ärzte mit der Situation konfrontiert waren, dass unverzichtbare Arzneimittel nicht oder nur einge- schränkt zur Verfügung standen. Wir sollten im Sinne unserer Patientinnen und Patienten alles dafür tun, dass in der Bundesrepublik Deutschland mit ihrem exzellenten Gesundheitssystem so etwas nicht mehr an der Tagesordnung ist."

Die im Rahmen der Pressekonferenz vorgestellte Aktualisierung und der 9. Band der Gesundheitspolitischen Schriftenreihe der DGHO „Arzneimittelengpässe am Beispiel der Hämatologie und Onkologie "können heruntergeladen werden unter: https://www.dgho.de/publikationen/ schriftenreihen/arzneimittelengpaesse.

Die Aufzeichnung der Pressekonferenz vom 26.11.2019 ist unter folgendem Link abrufbar: https:// youtu.be/lw3OHJ6zCeM. 